\title{
Static Summarization of Video Scenes Based on Minimal Spanning Tree
}

\author{
Partha Pratim Mohanta ${ }^{1}$, Sudipta Chowdhury ${ }^{2}$, Arnab Roy ${ }^{2}$, \\ Sanjoy Kumar Saha ${ }^{2}$, and Bhabatosh Chanda ${ }^{1}$ \\ 1 ECS Unit, Indian Statistical Institue, Kolkata, India \\ ${ }^{2}$ CSE Dept., Jadavpur University, Kolkata, India
}

\begin{abstract}
The common practice for providing a static summarized view of a video is to create a storyboard. Storyboard is the chronological arrangement of the representative frames. Shot level storyboard suffers from redundancy as in a scene constituting shots normally repeat. Also the size of such storyboard is a constraint for many application. In this work we have considered scene as the more meaningful unit. We propose a state-based scene segmentation algorithm and also a minimal spanning tree based novel method to select the representative frames for the scenes. Storyboard consisting of scene level representative frames are much more compact than shot level storyboard. Moreover, scene being the semantic unit, flow of semantic content of the video data is well preserved. Experimental result confirms the claim.
\end{abstract}

\section{Introduction}

The huge volume is the major challenge in working with video data. As a result efficient browsing, indexing and summarization of video data has emerged as an active area of research in the recent years. For all such application, video segmentation acts as a fundamental step. Video segmentation is the process to partition the video into meaningful units. Shot is the basic structural unit and it is a sequence of frames captured through single operation of a camera. But for better understanding a video, a higher level semantic unit, called scene is formed. A scene is a collection of semantically related adjacent shots which depicts a high level concept.

The two major tasks for storyboard based summarization are video segmentation and thereafter determining the representative frames for the segmented units. In the context of video segmentation most of the efforts are directed towards temporal segmentation i.e. shot detection. Transition between the shots may be abrupt (cut) or gradual. A lot of works have been reported for shot level segmentation 112. Past study reveals that so far the summarized view of a video has been considered as a storyboard/ToC at the shot level. Semantically scene is the unit of the story conveyed by a video data. Ideally, the scene level storyboard can represent the content much better rather than shot level storyboard or a reduced version of it. A scene consist of contiguous shots that are semantically 
related, but may not be visually similar. Shots in a scene usually follow a quasiperiodic repetition. A heuristic approach based on the quasi-periodic pattern of the shots [3] is presented to detect the scenes in a video. Various Graph based schemes are also reported in 45 .

The next crucial task is to determine the representative frames (or key-frames) of shots. Determining the number of key-frames in a shot is an issue. Early approaches [6] considered first/last frame of the shot or both as representative. Mohanta et al. 8] have proposed a scheme that decomposes a shot into subshots with uniform visual content and from each such sub-shot one frame is taken as the representative. Using these key-frames a shot level storyboard may be generated in 9 . As the similar shots repeat in a scene, shot level storyboard bears substantial redundancy. Fuzzy-ART and Fuzzy C-Means based scheme 10] are also tried to minimize the redundancy. Mohanta et al. 8] have presented a spanning tree based scheme to prepare a size constrained storyboard.

Video summarization has many important applications including video-ondemand. Summarized video in the form of storyboard consisting of representative frames are most appealing and convincing compared to any textual description. Such summarization is most efficient and effective if it is done at the scene level. in this work we summarize a given scene by a minimal subset of representative frames. These frames placed in chronological order forms the storyboard and also act as bookmark/table of content (ToC) enabling random access to the desired unit. Our main contribution in this work are two folds: first, we have modified algorithm presented in 3 for scene detection; second, is to map the classical MST algorithm in a novel way to divide the key-frames of a scene into visually homogeneous groups and also to extract representative frame from each of these groups. The present endeavor is a continuation of our earlier work on shot segmentation [2, key-frame extraction [8] and scene segmentation [3]. However, care is taken to design the proposed algorithm such that any error committed in the earlier stages does not affect the output much.

The paper is organized as follows. Following this introduction and a brief survey of the relevant work, a scene detection algorithm which is a modified version of our earlier work [3] is presented in section 2. Then, in the same section we present a methodology to find out the scene level representative frames which will be used in the storyboard. Experimental results are presented in section 3 and finally, concluding remarks are cited in section 4 .

\section{Proposed Methodology}

Scene based summarization is more meaningful where shots in a scene together represent an event in the context of the semantic content of the video. Although the shots may not be visually uniform but they jointly contribute to narrate the story in the scene. It has a strong resemblance with the human perception [11. Representative frames of the scenes may be organized to provide a summarized view/ToC at the scene level. Moreover, such a view is free from the redundancy present in the shot level storyboard and renders a more concise representation. 
The output of earlier schemes which work on shot level storyboard do not ensure the continuity in terms of the story depicted by the video. But, scene level storyboard ensures continuity of the events and also achieves conciseness. This dual advantage has motivated us to go for scene level summarization. The major steps of scene level summarization are as follows.

- Segment the video data

- Detect the shot boundaries

- Identify the representative frame(s) for each shot

- Detect the scene boundaries

- Identify the representative frames for each scene

- Organize the representative frames into storyboard

\subsection{Shot and Key-Frame Detection}

In our work, shot boundaries are detected following the methodology presented in 2. A unified model of shot boundary detection has been presented to deal with both abrupt and gradual transition. To detect the scene boundaries, each shot is first represented by one or more representative frames (key-frames). For each shot, key-frame(s) are selected using the methodology presented in 8]. In this work, Wald-Wolfowitz run (WWR) test is applied on each shot to examine its consistency in terms of visual content. The hypotheses of the WWR test are stated as follows:

$H_{0}: X$ and $Y$ are from same population, i.e., $F_{x}=F_{y}$

$H_{1}$ : They are from different population, i.e., $F_{x} \neq F_{y}$

where $X$ and $Y$ are two samples of sizes $m$ and $n$ respectively and the corresponding distributions are $F_{x}$ and $F_{y}$. Test statistic $W$ is computed based on $R$, the number of runs (a 'run' is a sequence of identical labels) as follows.

$$
W=\frac{R-\frac{2 m n}{N}-1}{\sqrt{\frac{2 m n(2 m n-N)}{N^{2}(N-1)}}}
$$

where $N=n+m$. Each frame is represented by a feature vector. Thus the multivariate version of the WWR test is used to detect the shots (sub-shots) which are visually consistent. For each such shot (sub-shot) one frame is taken as the representative based on the fidelity criteria.

\subsection{Scene Detection}

Shots in the video are represented by their key-frames and the video data becomes a sequence of key-frames. Scene boundaries are then detected by a modified technique of what is presented in [3]. For this we adopt the bag of words model [12. Key-frames are clustered and replaced by the corresponding cluster tag. Thus, the video data is converted into a sequence of tags. This sequence is then analyzed to detect the scene boundaries. Shots in a scene repeat themselves with different periodicity. Thus, a scene is a quasi-periodic sequence of 
shots. Based on this observation, concept of stable and quasi-stable state around which scenes are formed are defined. Finally, considering these states a heuristic algorithm is used to localize the scene boundaries as follows.

- Input: Let $T_{i}(i=1,2, \ldots, N)$ are the tags (or key-frames) representing the video, where $T_{i} \in\left\{t_{i} \mid i=1,2, \ldots, k\right\}, k$ is the number of cluster of key-frames and $k<<N$. Let $p$ be the length of the quasi-period at the $L$-th iteration.

- Step 1a: Any tag $T_{i}$ is marked Repetitive if there exists a tag $T_{j}$ within the span $i \pm p$ such that $T_{i}=T_{j}$.

- Step 1b: If the number of Repetitive tags is less than $m_{g} \times N\left(m_{g}<1\right)$, increase $p$ and repeat step 1a.

- Step 2: Suppose there exist two Repetitive tags $T_{i}$ and $T_{j}$ such that number of repetitive tags from $T_{i}$ to $T_{j}$ inclusive is more than $m_{l} \times|j-i|\left(m_{l}<1\right)$, we call this span of tags as stable state.

If a run of tags, which is not marked as stable state, is bounded by two stable states $\hat{S}_{1}$ and $\hat{S}_{2}$ and run length lies between $p$ and $2 p$, we call this span of tags as Quasi-stable state.

- Step 3a: If a quasi-stable state $Q$ is bounded by $\hat{S}_{1}$ and $\hat{S}_{2}$, and $\hat{S}_{1}$ and $\hat{S}_{2}$ possess some common tag, the $\hat{S}_{1}, Q$ and $\hat{S}_{2}$ are merged to a scene.

- Step 3b: If $3 \mathrm{a}$ is not true, and $Q$ and $\hat{S}_{1}$ possess some common tag then $\hat{S}_{1}$ and $Q$ merged to a scene.

- Step 3c: If $3 \mathrm{a}$ is not true, and $Q$ and $\hat{S}_{2}$ possess some common tag then $Q$ and $\hat{S}_{2}$ merged to a scene.

- Step 3d: If 3a, $3 \mathrm{~b}$ or $3 \mathrm{c}$ are not true, $Q$ is divided into two halves and each half is merged with adjacent $\hat{S}$ to form a scene.

- Step 4: If no more merging possible, feed each run of tags separately to step 1 to 3 as input with modified $N$ and $p$.

- Step 5: If $p=N$ and still some unmerged tags remain, declare every run of tags a scene.

\subsection{Selection of Representative Frames}

Once the scenes are detected, the representative frames for each scene have to be determined. Suppose, $C_{i}$ be the $i$-th scene of the video and $\tilde{F}_{i}$ be the set of all key-frames corresponding to the shots in the scene $C_{i}$. Thus, the task is to find out a $r f_{i} \subset \tilde{F}_{i}$ which can well represent $C_{i}$ and redundancy present in $\tilde{F}_{i}$ is also minimized substantially. In the proposed methodology we present a minimal spanning tree based scheme to which $\tilde{F}_{i}$ is provided as the input and $r f_{i}$ is obtained as the output. Each frame $f_{j} \in \tilde{F}_{i}$ is represented by $n$ dimensional feature vector $V_{j}=<v_{1}, v_{2}, \ldots, v_{n}>$ and treated as a node in the minimal spanning tree. To start with a node is taken as a root. Gradually, all the nodes are inserted into the tree. In each iteration from the remaining nodes, one nearest to the tree is included as the child of the nearest node in the tree. Distance between the child and parent is taken as the weight of the edge/link between them. The steps for preparing the tree are as follows.

- remaining_set $=$ set of all nodes(feature vector) 
- Choose randomly a node (feature vector), $n d_{i}$ as the root

- included_set $=n d_{i}$

- remaining_set= remaining_set - included_set

- Repeat the following steps until remaining_set is empty

- For each node, $n d_{r} \in$ remaining_set

* distance with the tree, $d_{k}=\min \left\{\operatorname{dist}\left(n d_{r}, n d_{i n}\right)\right\}$ where, $n d_{i n} \in$ included_set

- Let $d_{t}=\min \left\{d_{k}\right\}$ for the nodes $n d_{t r} \in$ remaining_set and $n d_{t i} \in$ included_set

- Put $n d_{t r}$ in the tree as child of $n d_{t i}$

- Set weight of the link between $n d_{t r}$ and $n d_{t i}$ as $d_{t}$

- Put $n d_{t r}$ in included_set

- Remove $n d_{t r}$ from remaining_set

In our experiment, each frame (node) in $\tilde{F}_{i}$ is represented by 512 dimensional color histogram. Each of the R, G, B plane of the frame is divided into 8 bins and normalized histogram is formed. Similarity between two nodes (i.e. feature vectors) is computed following Bhattacharyya distance. Distance between two nodes, $\operatorname{dist}\left(n d_{p}, n d_{q}\right)$ is taken as $1-$ bhattacharyya_distance $\left(V_{p}, V_{q}\right)$ where $V_{p}$ and $V_{q}$ are the normalized color histograms of the frames corresponding to $n d_{p}$ and $n d_{q}$ respectively.

To find out the representatives of the set of frames $\tilde{F}_{i}$, our target is to split the set into disjoint subsets where the elements in a subset are visually similar. Then one element from each subset is chosen as the representative based on certain criteria. Once the frames in $\tilde{F}_{i}$ are organized into minimal spanning tree $\left(m s t_{i}\right)$, the selection process of the representative frames for the scene $C_{i}$ proceeds as follows.

$-r f_{i}=\{\}$

- For each edge/link $e_{p q}$ in the minimal spanning tree, mst $_{i}$

- if (weight of $e_{p q}>t h$ ) then drop the link /edge

- for each subtree $s t_{j}$ in mst $_{i}$

- find the node $n d_{m}$ with maximum children

- $r f_{i}=r f_{i} \cup n d_{m}$

$-r f_{i}$ is the representative frames for the scene $C_{i}$

The minimal spanning tree, mst $_{i}$ is divided number of disjoint subtrees $\left\{s t_{j}\right\}$. Weight of a link/edge stands for the dissimilarity between the frames corresponding to a parent node and child node in $m_{s} t_{i}$. If it exceeds a threshold $(t h)$ then edge is removed. By removing such edges, mst $_{i}$ is splitted into number of disjoint subtrees where $s t_{j}$ consists of the nodes corresponding to visually similar frames in $\tilde{F}_{i}$. Finally, one representative from each subtree is chosen. The node with maximum children represents the frame having closest visual similarity with the majority frames in the subset and taken as the representative. In order to determine the value of $t h$, for each node (frame in $\tilde{F}_{i}$ ) distance with its nearest node (frame) is first determined. Let, for node $n d_{p}$ such distance is $d_{\text {min }_{p}}$. Then $t h$ is taken as $\mu_{i}+\sigma_{i}$ where $\mu_{i}$ and $\sigma_{i}$ stand for $\operatorname{avg}\left\{d_{\text {min }_{p}}\right\}$ and $\operatorname{std}_{-} d e v\left\{d_{\text {min }_{p}}\right\}$ 
respectively. Similar frames also reflect variation among themselves. A low value for $t h$ will put them into different subtrees. On the other hand, a high value may merge dissimilar frames also. The proposed value for $t h$ is a balanced one. $\mu_{i}+\sigma_{i}$ restricts the subtree to intrude into a dissimilar one but allows the subtree grow so that similar frames are included despite of variation among them.

\section{Experimental Result}

In order to carry out the experiment, we have worked with four movie videos namely Hirak Rajar Deshe (HRD) (a Satyajit Ray film), Mission Impossible-I (MI), Beautiful Mind (BM) and part of Catch Me If You Can (CMIYC). The details of the movies are shown in Table 1.

In our experiment shot and key-frames of a video are detected using the methodologies as described in section 2.1. Shot level storyboard is the arrangement of all the key-frames in order of their appearance in the video. Scene segmentation is performed by the algorithm presented in 2.2. Depending on the activity and dynamics present in the scene, it is a collection of different shots. Some of them are visually similar occurring at certain interval. Thus, shot level storyboard reflects lots of redundancy which are avoided in the scene level summary. Representative frames for the scenes are obtained following the methodology presented in the section 2.3. Chronological ordering of the same provides the summarized storyboard at scene level. Table 1 shows that in comparison to shot level storyboard, huge amount of size reduction is achieved in case of scene level summarization. Fig. 1 shows few examples where the key-frames of all the shots in a scene and representative frames of the scene are shown.

Table 1. Performance of summarization

\begin{tabular}{|c|c|c|c|c|c|c|}
\hline \multirow[t]{2}{*}{ Movie } & \multirow{2}{*}{$\begin{array}{c}\text { duration } \\
\text { (HH:MM:SS) }\end{array}$} & \multirow{2}{*}{ \# shots } & \multirow[t]{2}{*}{ \# scenes } & \multicolumn{2}{|c|}{ \# frames in summary } & \multirow{2}{*}{$\begin{array}{c}\% \text { of reduction in } \\
\text { scene level summary }\end{array}$} \\
\hline & & & & shot level & scene level & \\
\hline HRD & $01: 52: 27$ & 1331 & 62 & 1511 & 113 & 92.52 \\
\hline MI & $01: 45: 42$ & 1465 & 121 & 1582 & 228 & 85.59 \\
\hline$\overline{\mathrm{BM}}$ & $01: 55: 39$ & 1468 & 98 & 1768 & 172 & 89.93 \\
\hline CMIYC & $00: 36: 37$ & 312 & 32 & 427 & 48 & 88.76 \\
\hline Overall & $06: 10: 25$ & 4576 & 313 & 5288 & 561 & 89.39 \\
\hline
\end{tabular}

Evaluation of summary is a difficult task as it is quite subjective. In order to do so, shot level storyboard of the video is presented to the viewers. Scene level summary generated by the system is then made available to them. Every viewer then marks which of the scene level representatives generated by the system are irrelevant and frames in the shot level storyboard which are desirable but missing in the scene level summary. With these information, precision and recall are computed. In our effort, we have considered 10 viewers. Average precision and recall achieved by the proposed methodology are $92.88 \%$ and $88.01 \%$ respectively. 

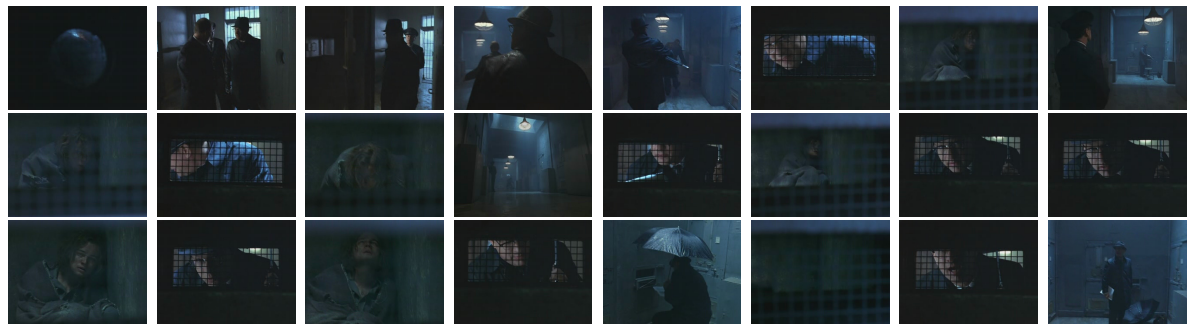

(i)(a) Key-frames of the shots in a scene from the movie Catch Me If You Can


(i)(b)Representatives for the scene shown in (i)(a)

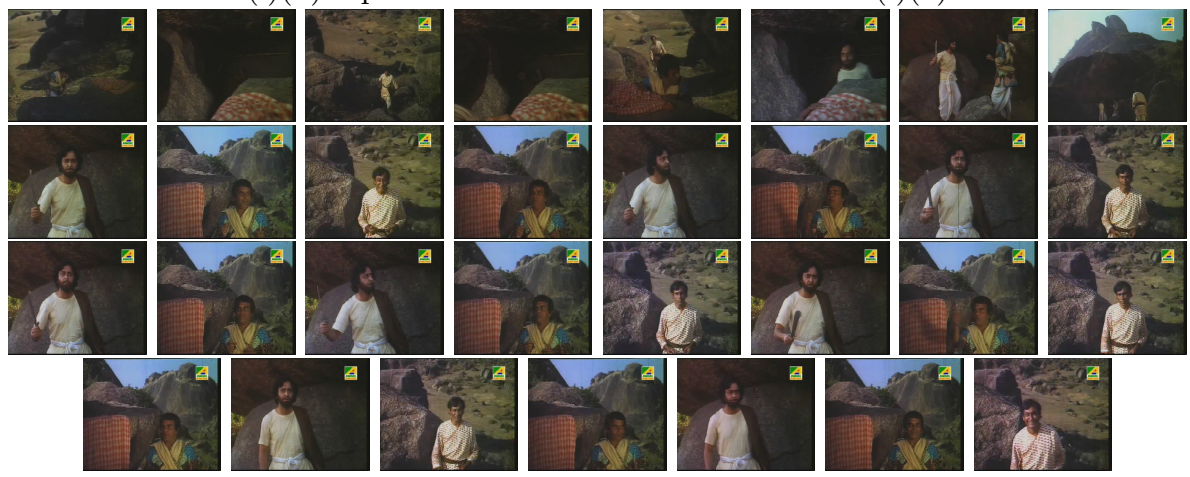

(ii)(a) Key-frames of the shots in a scene from the movie Hirak Rajar Deshe
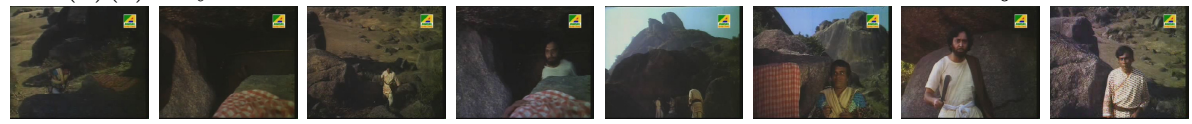

(ii)(b)Representatives for the scene shown in (ii)(a)
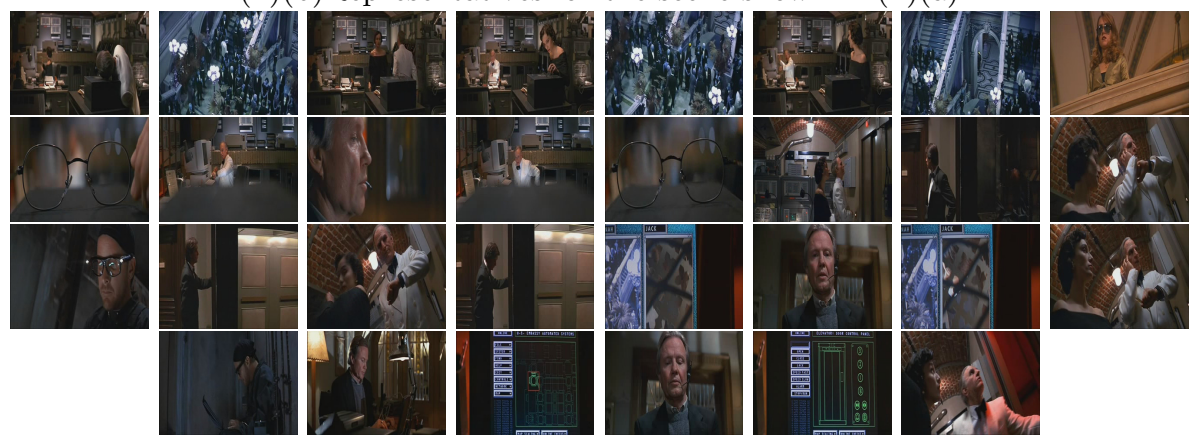

(iii)(a) Key-frames of the shots in a scene from the movie Mission Impossible-I
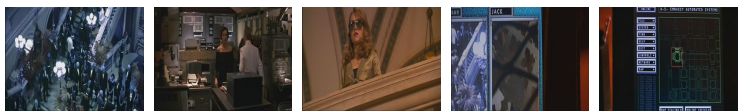

(iii)(b)Representatives for the scene shown in (iii)(a)

Fig. 1. Few examples of scene level summary for scenes from different movies 


\section{Conclusion}

In this work we have presented a state-based scene detection algorithm and a novel methodology to generate a scene level static summary of the video. In a shot level storyboard, key-frames of all the shots are presented chronologically. As similar shots appear periodically, the storyboard thus obtained possesses redundant frames. To get rid of it, we have considered scene as the semantic unit. A scene is a collection of consecutive shots of various types. A minimal spanning tree based scheme has been presented which determines the representative frames for the scene. Such representatives of all the scenes arranged chronologically renders the scene level static summary/storyboard. Experimental result indicates that the performance of the proposed methodology is satisfactory and achieves huge reduction in storyboard size in comparison to shot level summary.

\section{References}

1. Cernekova, Z., Pitas, I., Nikou, C.: Information theory-based shot cut/fade detection and video summarization. IEEE Trans. on CSVT 16(1), 82-91 (2006)

2. Mohanta, P.P., Saha, S.K., Chanda, B.: A model-based shot boundary detection technique using frame transition parameters. IEEE Transactions on Multimedia 14(1), 223-233 (2012)

3. Mohanta, P.P., Saha, S.K., Chanda, B.: A heuristic algorithm for video scene detection using shot cluster sequence analysis. In: Proc. ICVGIP, India (2010)

4. Rasheed, Z., Shah, M.: Detection and representation of scenes in video. IEEE Trans. on Multimedia 7(6), 1097-1105 (2005)

5. Zhao, Y.J., Wang, T.: Scene segmentation and categorization using n-cuts. In: Proc. CVPR, pp. 343-348 (2007)

6. Tonomura, Y., Akutsu, A., Otsugi, K., Sadakata, T.: Videomap and videospaceicon: Tools for automatizing video content. In: Proc. ACM INTERCHI, pp. 131-141 (1993)

7. Rui, Y., Huang, T.S., Mehrotra, S.: Exploring video structures beyond the shots. In: Proc. IEEE Conf. on Multimedia Computing and Systems, pp. 237-240 (1998)

8. Mohanta, P.P., Saha, S.K., Chanda, B.: A novel technique for size constrained video storyboard generation using statistical run test and spanning tree. International Journal of Image and Graphics 13(1), 1-24 (2013)

9. Macer, P.J., Thomas, P.J.: Video storyboards: Summarizing video sequences for indexing and searching of video databases. In: Proc. IEE Colloquium on Intelligent Image Databases, pp. 2/1-2/5 (1996)

10. Cayllahua-Cahuina, E.J.Y., Camara-Chavez, G., Menotti, D.: Video summarization with automatic shot detection using color histograms. In: Proc. IPCV 2012, Las Vegas, USA (2012)

11. Rui, Y., Huang, T.S., Mehrotra, S.: Constructing table-of-content for video. ACM Multimedia Systems 7(5), 359-368 (1999)

12. Sivic, J., Zisserman, A.: Efficient visual search of videos cast as text retrieval. IEEE Trans. on Pattern Analysis and Machine Intelligence 31(4), 591-606 (2009) 